OPEN ACCESS

Edited by:

Dario Coletti,

Sapienza University of Rome, Italy

Reviewed by:

Jennifer Stevenson Moylan, University of Kentucky, United States Emanuele Berardi,

VIB-KU Leuven Center for Cancer Biology, Belgium

*Correspondence:

Rosanna Piccirillo rosanna.piccirillo@marionegri.it

Specialty section: This article was submitted to

Striated Muscle Physiology, a section of the journal Frontiers in Physiology

Received: 21 December 2018 Accepted: 04 March 2019

Published: 29 March 2019

Citation:

Piccirillo R (2019) Exercise-Induced Myokines With Therapeutic Potential for Muscle Wasting. Front. Physiol. 10:287. doi: 10.3389/fphys.2019.00287

\section{Exercise-Induced Myokines With Therapeutic Potential for Muscle Wasting}

\author{
Rosanna Piccirillo* \\ Department of Neurosciences, Mario Negri Institute for Pharmacological Research IRCCS, Milan, Italy
}

Skeletal muscle is a highly vascularized tissue that can secrete proteins called myokines. These muscle-secreted factors exert biological functions in muscle itself (autocrine effect) or on short- or long-distant organs (paracrine/endocrine effects) and control processes such as metabolism, angiogenesis, or inflammation. Widely differing diseases ranging from genetic myopathies to cancers are emerging as causing dysregulated secretion of myokines from skeletal muscles. Myokines are also involved in the control of muscle size and may be important to be restored to normal levels to alleviate muscle wasting in various conditions, such as cancer, untreated diabetes, chronic obstructive pulmonary disease, aging, or heart failure. Interestingly, many myokines are induced by exercise (muscle-derived exerkines) and some even by specific types of physical activity, but more studies are needed on this issue. Most exercise-induced myokines travel throughout the body by means of extracellular vesicles. Restoring myokines by physical activity may be added to the list of mechanisms by which exercise exerts preventative or curative effects against a large number of diseases, including the deleterious muscle wasting they may cause. Extending our understanding about which myokines could be usefully restored in certain diseases might help in prescribing more tailored exercise or myokine-based drugs.

Keywords: myokines, exercise, exerkines, skeletal muscles, atrophy

\section{INTRODUCTION}

Skeletal muscle is the largest tissue in the human body, accounting for about $30 \%$ of body mass in women and $40 \%$ in men. Although, it can suffer in many incurable diseases, it is unexpectedly the most "undrugged" tissue. Skeletal muscle is very plastic because it can be enlarged with adaptation to specific exercise and/or high-protein content diet or reduced in catabolic conditions like fasting, cancer, untreated diabetes, heart failure, AIDS, chronic obstructive pulmonary disease (COPD), or aging (Piccirillo et al., 2014; Furrer and Handschin, 2018). This tissue is the main body's protein reservoir, able to break down its own proteins and release aminoacids into the bloodstream during stress or fasting or disease. This process goes under the name of muscle wasting or cachexia, and ultimately aggravates the disease state, even leading individuals to death (Kalantar-Zadeh et al., 2013). Losing more than about $40 \%$ of normal body mass was incompatible with life in starved, lager-confined Jews (Winick, 1979) and in HIV-infected patients before the successful advent of antiretroviral therapy (Kotler et al., 1989; Roubenoff and Kehayias, 1991). 
Reduction in muscle size mainly consists of a decrease in cell size caused by loss of organelles, cytosol and proteins. This deleterious process is mainly driven by increased protein breakdown through enhanced proteasomal and lysosomal activities (coordinatively activated by FoxO3-dependent transcription) (Sandri et al., 2004; Zhao et al., 2007) and reduced protein synthesis (mainly regulated by the IGF-1/PI3K/AKT pathway). During muscle atrophy, the removal of ATP-producing organelles, such as mitochondria, through enhanced autophagy (i.e., mitophagy) explains the increased propensity to fatigue of muscle-losing patients (VanderVeen et al., 2017).

Physical exercise is among the ways by which muscles may be protected against disease-induced muscle wasting. Physical activity can be grossly divided into two types: aerobic (or endurance) and anaerobic (or strength) exercise. The former shifts muscle fiber types toward those with increased capacity for aerobic metabolism and better ability to resist fatigue, due to a larger number of mitochondria and vessels (types I and IIA fibers), this is typical in runners, long-distance cross-country skiers, bicyclists, swimmers. These fibers contract slowly with a low peak force and generate ATP through oxidative phosphorylation of glucose and non-esterified fatty acids. The second type of exercise causes hypertrophy, especially of myofibers IIX (and IIB in rodents), due to enhanced synthesis of contractile proteins, resulting in increased strength with no change in the number of mitochondria (typically occurring in weight-lifters and body-builders). These fibers generate fast contractions with a high peak force, and metabolize phosphocreatine and glucose anaerobically to make ATP (Flück and Hoppeler, 2003; Hawley et al., 2014).

Aerobic exercise induces in humans (Pilegaard et al., 2003) and in rodents (Terada et al., 2002) the peroxisome proliferatoractivated receptor $\gamma$ coactivator $1-\alpha(P G C 1-\alpha)$. PGC1- $\alpha$ not only promotes mitochondrial biogenesis, contrasting the propensity to fatigue of cachectic muscles, but also directly antagonizes protein catabolism by blocking FoxO3 (Sandri et al., 2006), the master transcription factor coordinating both proteasomal and lysosomal protein degradation (Sandri et al., 2004; Mammucari et al., 2007; Zhao et al., 2007). As a result, oxidative fibers are more resistant to cancer-induced atrophy than glycolytic ones, typically enlarged upon muscle adaptation to anaerobic exercise (Ciciliot et al., 2013). Conversely, anaerobic exercise, by stimulating mainly myofibrillar protein synthesis through activation of the PI3K/AKT pathway and overproduction of IGF1 (McCall et al., 2003), may obviate muscle wasting by enhancing protein synthesis and inactivating FoxO3 through AKT-mediated phosphorylation (Sandri et al., 2004).

It is becoming clear that physical activity mediates the release from muscles of factors with anti-atrophic effects in an autocrine fashion but, surprisingly, these muscle-derived molecules can even contrast the primary disease, as is the case of the recently identified anti-tumoral molecules of muscle origin (Aoi et al., 2013; Gannon et al., 2015). Some examples are oncostatin M, able to restrain mammary cancer cell growth in vitro (Hojman et al., 2011), fatigue substance (F-Substance) isolated from muscles of trained rats and displaying inhibitory effect on the breast cancer cell line MCF-7 (Munoz et al., 2013) and secreted protein acidic and rich in cysteine (SPARC). The latter is induced in plasma of trained individuals or mice and it is able to suppress colon tumorigenesis via regular exercise in mice (Aoi et al., 2013).

Skeletal muscle is a highly vascularized tissue and has secretory abilities (Pedersen and Febbraio, 2008). In fact, muscles release not only aminoacids in response to increased energy demand and fuel the liver to undergo gluconeogenesis, but also proteins to mediate inter-tissue crosstalk. These molecules have been named myokines to underline their muscle origin. The current definition of myokines is "cytokines or peptides which are secreted by skeletal muscle cells and subsequently released into the circulation to exert endocrine or paracrine effects in other cells, tissues or organs" (Pedersen and Febbraio, 2012). Not all myokines are exclusively originating by skeletal muscles. Some myokines are mainly muscle-restricted proteins, like myostatin, while others can also be secreted by other tissues, as is the case of the adipomyokines (for example, IL-8 and MCP-1) (Trayhurn et al., 2011). However, skeletal muscle is probably the main source of most myokines secreted also by other tissues in the circulation because it is amply vascularized and makes up $30-40 \%$ of the human body mass.

More than 3000 myokines have been reported (Whitham and Febbraio, 2016) and, among them, it is worth mentioning those identified in humans: angiopoietin-like 4 (ANGPTL4), apelin, brain-derived neurotrophic factor (BDNF), CCL2 or MCP-1, CX3CL1 of fractalkine (FKN), fibroblast growth factor 21 (FGF21), interleukin-6 (IL-6), IL-7, IL-8, IL-15, irisin, leukemia inhibitory factor (LIF), meteorin-like protein (Metrnl), myostatin and SPARC (for a review see Catoire and Kersten, 2015). Myokine secretion is a process conserved among species: Drosophila melanogaster (Zhao and Karpac, 2017), adult Danio rerio (i.e., Zebrafish) (Rovira et al., 2017), and mammals (Demontis et al., 2013b), among others, all have muscles able to release such factors. The first reported myokine, Interleukin-6 (IL-6), is conserved in Drosophila melanogaster where three orthologs exist, Unpaired (Upd) also called Outstretched, Upd2 and Upd3, increases with exercise (Ostrowski et al., 2000) and has antiinflammatory properties, at least in mammals (Pedersen and Febbraio, 2008). Another example is myostatin that is secreted by skeletal muscles and can stimulate activin type II receptors (ActRII), ultimately leading to muscle atrophy; it is conserved in Drosophila melanogaster (myoglianin) and in Zebrafish (myostatin b). Notably, myostatin inactivation by spontaneous missense mutations or intentional genetic ablation results in hypermuscularity in a large number of species (McPherron and Lee, 1997; McPherron et al., 1997; Prontera et al., 2009).

Some myokines are preferentially secreted by glycolytic fibers [e.g., musclin (Banzet et al., 2007; Subbotina et al., 2015), osteoprotegerin and angiogenin (Rutti et al., 2018)], and others by oxidative fibers [e.g., myonectin (Seldin et al., 2012)] but the fiber-type preference, if it exists, is unknown for most of the other myokines. Along the same line, FGF21 that is an hormonelike molecule involved in metabolism has been described as an AKT-regulated myokine (Izumiya et al., 2008a; He et al., 2018), hence, more typically secreted by glycolytic fibers, while irisin expression is induced by PGC1 $\alpha$, thus, more typically secreted by oxidative ones (Boström et al., 2012). Some myokines have been renamed muscle-derived exerkines (Safdar and Tarnopolsky, 2018) because they are induced by physical activity and - most 
interestingly - some of them show a preference for muscle induction through specific types of physical exercise (Figure 1A) (Kanzleiter et al., 2014; Bazgir et al., 2015; Shin et al., 2015; Laker et al., 2017; Ishiuchi et al., 2018).
Lecker et al. (2004) found a subset of genes that were differentially expressed in muscles undergoing wasting in unrelated conditions (fasting, uremia, tumor, and diabetes) in rodent models and named them "atrogenes." Among them,

A

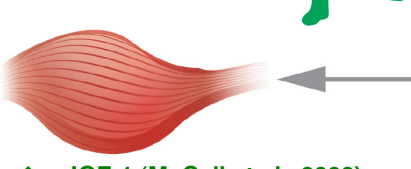

$\uparrow$ IGF-1 (McCall et al., 2003)

Myostatin (Walker et al., 2004)

$\uparrow$ Decorin (Kanzleiter et al., 2014)

$\uparrow$ IL-15 (Bazgir et al., 2015)

$\uparrow$ Irisin (Kim et al., 2015)

^ BMP7 (Kim et al., 2016)

$\uparrow$ Angiopoietin-like 4 or ANGPL4 (Laker et al., 2017)

FGF21 (He et al., 2018)
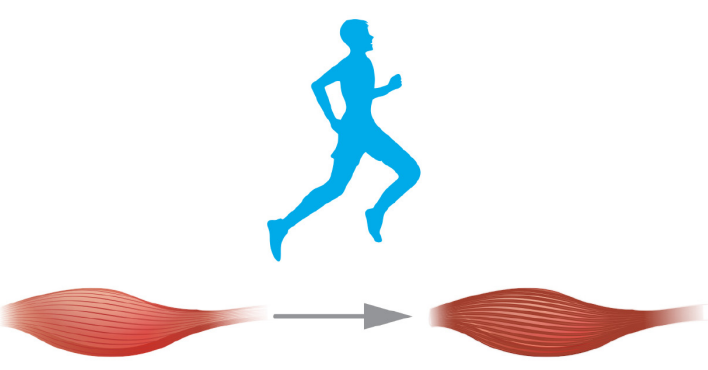

$\uparrow \quad$ IL-6 (Ostrowski et al., 1998)

$\uparrow \quad$ LIF (Broholm et al., 2008)

$\uparrow \operatorname{VEGF}$ (Arany et al., 2008)

$\downarrow$ Myostatin (Hittel et al., 2010)

$\uparrow \quad$ Irisin (Boström et al., 2012)

$\uparrow$ Myonectin (Seldin et al., 2012)

$\uparrow$ SPARC (Aoi et al., 2013)

$\uparrow$ Apelin (Besse-Patin et al., 2014)

$\uparrow$ CCL2 or MCP-1 (Catoire et al., 2014)

$\uparrow$ CX3CL1 or fractalkine or FKN

(Catoire et al., 2014)

$\uparrow \quad$ IL-15 (Shin et al., 2015)

$\uparrow$ Musclin (Subbotina et al., 2015)

$\uparrow$ SDF1 (Puchert et al., 2016)

$\uparrow$ FGF21 (Tanimura et al., 2016)

$\Downarrow \quad$ CCL5/RANTES (Ishiuchi et al., 2018)

B

$\uparrow$ IL=8 (Amir Levy et at., 2015)

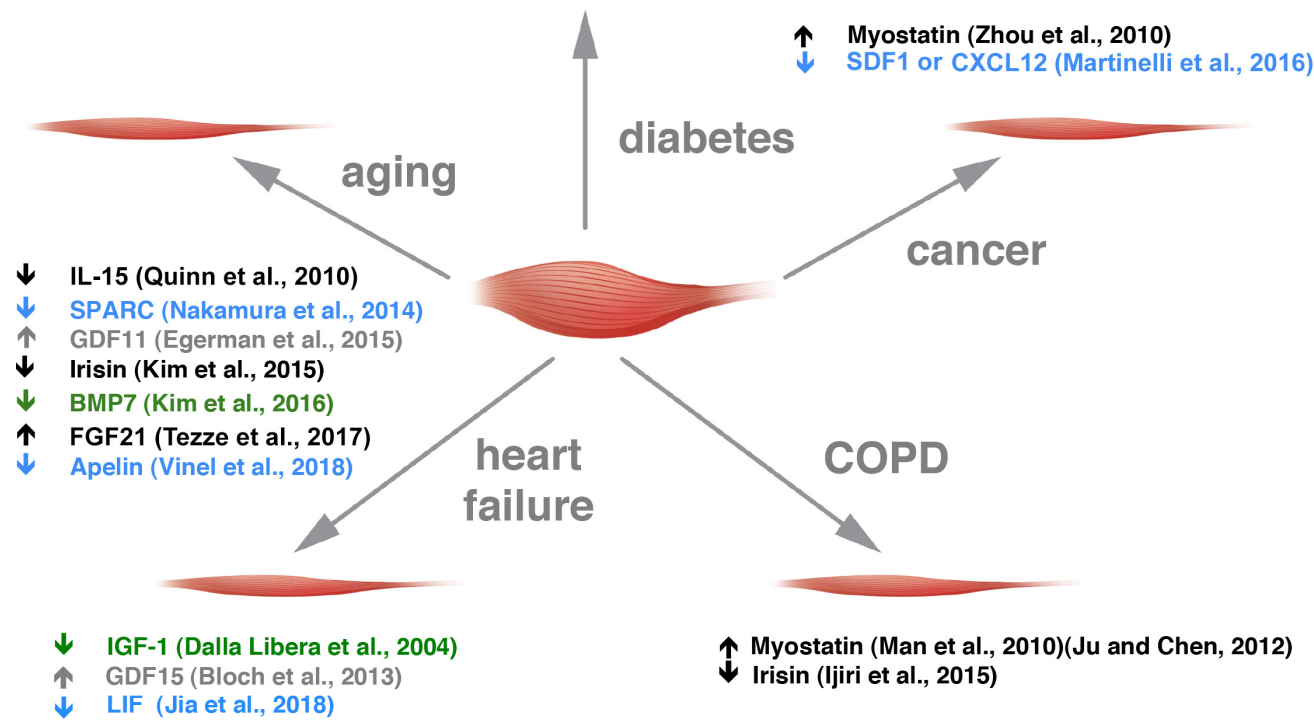

FIGURE 1 | Exercise-sensitive myokines with a preference for aerobic or anaerobic exercise are altered in different muscle wasting disorders. (A) Muscle adaptation to anaerobic and aerobic exercise is grossly depicted. In green, myokines altered in anaerobic exercise are listed with the appropriate references. In blue, those altered by endurance training are reported. (B) Myokine alterations in the five diseases indicated are listed. Myokines sensitive to anaerobic or aerobic exercise are depicted in green and blue, respectively, and those that change in both kinds of exercise in black. Myokines whose alteration by exercise is not clear are indicated in gray. 
a subset of potentially secreted proteins, called "extracellular matrix proteins," were all downregulated in all conditions of muscle atrophy tested and included fibrillin 1, fibronectin 1, galectin 1 , osteoblast specific factor 2 , secreted modular calciumbinding protein 2, and insulin-like growth factor binding protein 5. It would be worth clarifying whether these include some that could be useful to reverse to basal levels to contrast atrophy, and whether they can be classified as myokines or not (i.e., "atromyokines").

Most exercise-induced myokines travel through the body by means of exosomes measuring between 30 and $100 \mathrm{~nm}$ in diameter, or micro- or nano-sized extracellular vesicles (EVs), through analyses in Vesciclepedia and ExoCarta (Safdar and Tarnopolsky, 2018). These muscle-derived EVs increase in number in the bloodstream upon exercise, perhaps through calcium-dependent secretion coupled with muscle contractions (Whitham et al., 2018). In a very elegant work, Whitham et al. (2018) showed that when EV liberated in the blood of healthy individuals after $1 \mathrm{~h}$ bout of cycling exercise were labeled and transferred to donor mice, they were able to target organs like the liver, but not all organs have been scrutinized. The search for exercise factors of muscle origin mediated by these structures has just started and could help explain why myokines are not rapidly degraded by the numerous proteases in the extracellular environment. Their protective lipid bilayers could facilitate them to target muscle-unrelated tissues, affecting overall body homeostasis. This would further help explain how genetic modifications restricted to muscles impact on other tissues (Rai and Demontis, 2016) as the reduced adiposity reported in animals with muscle deficiency of myostatin (McPherron and Lee, 2002) or the increased resistance to diet-induced obesity and steatosis of mice with muscles overexpressing AKT1 (Izumiya et al., 2008b).

The secretion of myokines is altered in a growing number of diseases, including congenital myotonic dystrophy (Nakamori et al., 2017), and modulable through physical activities (Benatti and Pedersen, 2015). This minireview gives an overview of such factors in health and muscle atrophy-causing diseases (Figure 1B) and how physical exercise could correct some of them toward healthier levels, to spare muscle mass.

\section{MYOKINES ALTERED IN DIABETES}

Tissue cross-talk is emerging as vital to coordinate the different organs implicated in glucose homeostasis. Among the crosstalking factors, muscle-secreted myokines can influence the function and survival of pancreatic $\beta$-cells as is the case of angiogenin (previously known as an angiogenesis-promoting factor) (Gao and $\mathrm{Xu}, 2008$ ) and osteoprotegerin (involved also in bone density's regulation) (Simonet et al., 1997) that protect $\beta$ cells from the deleterious effects of proinflammatory cytokines (Rutti et al., 2018).

Diabetes accounts for about $15 \%$ of total diseases related to selected myokines (apelin, BDNF, IL-15, irisin, SPARC) (Son et al., 2018). In adult humans, an insulin-resistant state preceding diabetes onset, already alters myokine secretion, as is the case of increased release of myonectin, but not FGF21 or myostatin
(Toloza et al., 2018), but the biological effects of this increase are not clear yet. In another study, FGF21 was found increased in the plasma of diabetic individuals, but if this is a compensatory mechanism to increase glucose uptake from muscles remains to be explored (Mashili et al., 2011). This myokine was found increased also in human and murine plasma after a single bout of endurance exercise (Tanimura et al., 2016). In streptozotocininduced diabetic mice, myostatin is increased and causes muscle wasting (Hulmi et al., 2012). Molecules able to neutralize this myokine are under tests for the treatment of diabetic myopathy in animal models, but with contradictory results so far (Wang et al., 2015; Dong et al., 2016).

Puzzingly, both CX3CL1 (Shah et al., 2011) and Metrln (Chung et al., 2018) were elevated in the blood of patients afflicted by type II diabetes and also in plasma and/or muscles of individuals after acute endurance exercise (Catoire et al., 2014) or short-term interval training (Eaton et al., 2018), but so far their possible contribution to muscle size control has not been explored. Finally, increased plasma apelin has been found to predict type II diabetes but only in men, for reasons we still do not understand (Ma et al., 2014). While the role of apelin in improving insulin resistance by enhancing glucose utilization by muscle and fat is clear (Dray et al., 2008), its direct ability to protect muscles from diabetes-induced atrophy deserves further studies, but its elevated expression upon aerobic exercise is of note (Besse-Patin et al., 2014). IL-8, a chemokine whose muscle secretion is altered by exercise in humans, though mostly after extreme conditions in response to an ultraendurance exercise bout in experienced athletes (Marklund et al., 2013), was found to be overproduced by myotubes from type II diabetes patients and to disfavor muscle capillarization, reducing muscle exposure to glucose, so possibly further impinging on muscle wasting and the primary disease itself (Amir Levy et al., 2015).

Further studies are needed to see whether and which other myokine(s) is/are altered in muscles undergoing diabetic atrophy (for a further summary on this topic see Carson, 2017), and whether it might be useful to target for the cure of this kind of atrophy.

\section{MYOKINES ALTERED IN CANCER CACHEXIA}

Muscle wasting occurs in $80 \%$ of patients with advanced cancer and causes death in 30-40\% of cases (Fearon et al., 2011). Various myokines may be involved in this kind of atrophy (for a review see Manole et al., 2018). Cancer accounts for only 3.3\% of total diseases related to selected myokines (apelin, BDNF, IL-15, irisin, SPARC) (Son et al., 2018), but additional myokines have been shown to be more clearly involved in cancer cachexia.

We found stromal derived factor 1 (SDF1), also referred to as CXCL12, as specifically and strongly downregulated only in skeletal muscles of tumor-bearing mice and not in those undergoing wasting for other reasons (i.e., uremia, diabetes, fasting, or disuse) (Martinelli et al., 2016). Overexpression of SDF1 in muscle through in vivo electroporation of plasmid spared not only the cross-sectional area of transfected fibers but also that of adjacent untransfected ones, indicating that 
SDF1 also exerts its anti-atrophic effects in a paracrine fashion (Martinelli et al., 2016). Interestingly, Puchert and coworkers found it to be induced in muscles of mice trained by running for 4 weeks (Puchert et al., 2016). This muscle-secreted factor has a role in myogenesis, muscle regeneration (Bobadilla et al., 2014) and angiogenesis, similarly to VEGF (Arany et al., 2008) and could be involved in increased muscle capillarization upon adaptation to aerobic exercise. Overall, elevated muscle secretion of SDF1 could be ascribed to the mechanisms by which aerobic exercise or some drugs (e.g., Sunitinib) alleviate cancer-associated muscle wasting (Pretto et al., 2015).

Myostatin-based signaling was found increased in muscles of Yoshida hepatoma-bearing rats (Costelli et al., 2008), in C26-bearing mice (Zhou et al., 2010), and in patients with various malignancies (for a review see Han et al., 2013), while in pre-cachectic cancer patients circulating levels of myostatin increased only with certain types of tumors (Aversa et al., 2012). Importantly, while myostatin is the main factor limiting muscle size in mice, it seems that in humans activin A and growth differentiation factor 11 (GDF11) also play major roles and could be targeted to spare muscles during human diseases (LachTrifilieff et al., 2014). Anti-ActRII drugs have in fact been tested in cancer patients but unfortunately, there were no improvements in functional parameters (for a review see Furrer and Handschin, 2018). Myostatin is one of the few myokines whose secretion is reduced with both anaerobic (Walker et al., 2004) and aerobic exercise (Hittel et al., 2010), in concordance with its role as a negative modulator of muscle size.

The type of exercise that seems most effective at sparing muscles during cancer seems the aerobic and not the anaerobic one. In fact, the $\mathrm{AKT} / \mathrm{mTOR}$ signaling appears even hyperactivated in muscles of tumor-bearing rodents (Penna et al., 2010). So, future research should focus on understanding which aerobic exercise-induced myokine(s), besides SDF1, have anticatabolic or proanabolic action in muscles during cancer cachexia.

\section{MYOKINES ALTERED IN COPD}

Chronic obstructive pulmonary disease is an inexorable lung disease caused by excessive inflammation and subsequent damage to the airways and lung tissue, mainly occurring after long-term exposure to cigarette smoking (Passey et al., 2016).

Very few myokines have been studied in relation to COPD-induced muscle wasting, even though COPD is the most prevalent disease worldwide causing excessive myopenia and death (Lozano et al., 2012). COPD accounts for about $5 \%$ of total diseases related to selected myokines (apelin, BDNF, IL-15, irisin, SPARC) (Son et al., 2018). Consistent with its role in reducing muscle mass, myostatin expression is increased in the vastus lateralis (Plant et al., 2010) and quadriceps (Man et al., 2010) from COPD patients. Circulating myostatin protein is also elevated in serum from COPD patients and correlates with reduced muscle mass in males (Ju and Chen, 2012). Clinical trials to test bimagrumab (anti-ActRII-based drug) in COPD patients have recently showed increases in lean body mass and muscle volume in treated patients but unfortunately, no improvements in functional parameters (for a review see, Furrer and Handschin, 2018).

Irisin, a prominent PGC1 $\alpha$-induced myokine, is the cleaved product of the transmembrane protein fibronectin type III domain-containing protein 5 (FNDC5) (Boström et al., 2012) and is induced by resistance exercise in mice and humans (Kim et al., 2015). Most importantly, circulating irisin was low in COPD patients and its levels correlate with exercise capacity (Ijiri et al., 2015). More studies are still needed to dissect the mechanisms behind COPD-related muscle atrophy, because efficacious therapies are lacking.

\section{MYOKINES ALTERED IN AGING}

Many alterations occur in skeletal muscle with aging (Demontis et al., 2013a), including enhanced activity of the proteasomal pathway causing muscle atrophy (i.e., sarcopenia) (Altun et al., 2010), but the question whether muscle's ability to secrete myokines changes with aging has only recently started to attract attention. The increased sarcolemmal permeability of aged muscles may favor increased release of myokines during aging. Nonetheless, the expression of bone morphogenetic factor 7 (BMP7) is low in muscles of aged rats but can be restored through either uphill or gradual downhill running (Kim et al., 2016). This myokine may act by stabilizing the neuromuscular junction (NMJ) that is reduced in number and function with aging (for a review see, Demontis et al., 2013b; Larsson et al., 2019). Moreover, BMP7 has been found to cause muscle hypertrophy in mice through Smad1/5-mediated activation of mTOR signaling (Winbanks et al., 2013). Similar results have been obtained for irisin, whose secretion decreases in aging mice and is restored with resistance exercise training (Kim et al., 2015). While myostatin was unchanged in the elderly (Welle et al., 2002), another member of the same transforming growth factor $\beta$ (TGF $\beta$ ) family, GDF11, increases in blood (and muscles) during aging in rats and humans and, even more importantly, inhibits muscle regeneration (Egerman et al., 2015). The secretion of FGF21 also seems dysregulated in aged mice (Tezze et al., 2017).

Apelin is an exerkine that has been recently found to have a major role in counteracting age-associated muscle wasting (Vinel et al., 2018), also by promoting mitochondriogenesis, alleviating muscle-related inflammation and stimulating its regenerative capacity. Its possible role in disease-associated muscle atrophy is still unclear. SPARC is induced in plasma of humans by a single bout of steady-state cycling exercise at $70 \%$ of $\mathrm{VO}_{2}$ max for $30 \mathrm{~min}$, or after 4 weeks of cycling, three times a week for $30 \mathrm{~min}$ at $70 \%$ of $\mathrm{VO}_{2} \mathrm{max}$, and similarly in exercising mice (Aoi et al., 2013). This protein was low in aged muscle because its internalization in skeletal muscle progenitors becomes defective with age, so its anti-adipogenic effect could be limited and may contribute to fat infiltration in muscle upon aging (Nakamura et al., 2014).

Finally, IL-15 and the soluble form of its receptor also decrease in serum of aged mice (Quinn et al., 2010). These findings are in concordance with the single nucleotide polymorphisms (SNP) found in the human gene for such 
receptor that affects the muscularity and exercise capacity of individuals (Pistilli et al., 2008) and may influence interindividual propensity to sarcopenia.

\section{MYOKINES ALTERED IN HEART FAILURE}

Muscle wasting in patients with chronic heart failure (HF) identifies those at high risk of death (Anker et al., 1997). IGF1 decreases in the skeletal muscle of pre-cachectic HF patients (Hambrecht et al., 2002) and in animal models. Treatment with IGF-1 helped contrast the HF-induced muscle atrophy, improving exercise capacity (Dalla Libera et al., 2004).

GDF15 (also known as macrophage-inhibiting factor-1 and involved in appetite regulation) was found elevated in the acute muscle wasting following cardiac surgery and, interestingly, GDF15-treated myotubes undergo wasting, supporting a procachectic role of this factor (Bloch et al., 2013). A recent study showed the effect on muscle expression of an IL-6-like cytokine, LIF, in rats undergoing myocardial infarction. In this model the myocardial infarction-associated muscle wasting was recovered upon interval exercise and LIF was upregulated as well in gastrocnemii (Jia et al., 2018). These results contrast with other reports of a pro-cachectic role of (tumor-derived) LIF in cancerassociated muscle wasting (Seto et al., 2015), so further studies are needed to dissect the signaling pathway activated by LIF in muscle fibers and verify whether endogenous expression of LIF is altered in muscles atrophying because of various conditions. The upregulated expression of LIF by exercise is confirmed by other studies (Broholm et al., 2008) and further proven by the existence in the human LIF promoter of two nuclear factor of activated $\mathrm{T}$ cells (NFAT) binding sites (Bamberger et al., 2004), a transcription factor well-known to be activated by muscle contractions through the $\mathrm{Ca}^{2+}$ calmodulin-calcineurin axis (Schiaffino and Serrano, 2002).

Searching for similar binding sites in the gene regulatory regions of other putatively exercise-induced myokines should be

\section{REFERENCES}

Altun, M., Besche, H. C., Overkleeft, H. S., Piccirillo, R., Edelmann, M. J., Kessler, B. M., et al. (2010). Muscle wasting in aged, sarcopenic rats is associated with enhanced activity of the ubiquitin proteasome pathway. J. Biol. Chem. 285, 39597-39608. doi: 10.1074/jbc.M110.129718

Amir Levy, Y., Ciaraldi, T. P., Mudaliar, S. R., Phillips, S. A., and Henry, R. R. (2015). Excessive secretion of IL-8 by skeletal muscle in type 2 diabetes impairs tube growth: potential role of PI3K and the Tie2 receptor. Am. J. Physiol. Endocrinol. Metab. 309, E22-E34. doi: 10.1152/ajpendo.00 513.2014

Anker, S. D., Ponikowski, P., Varney, S., Chua, T. P., Clark, A. L., Webb-Peploe, K. M., et al. (1997). Wasting as independent risk factor for mortality in chronic heart failure. Lancet 349, 1050-1053. doi: 10.1016/S0140-6736(96)07015-8

Aoi, W., Naito, Y., Takagi, T., Tanimura, Y., Takanami, Y., Kawai, Y., et al. (2013). A novel myokine, secreted protein acidic and rich in cysteine (SPARC), suppresses colon tumorigenesis via regular exercise. Gut 62, 882-889. doi: 10. 1136/gutjnl-2011-300776

Arany, Z., Foo, S.-Y., Ma, Y., Ruas, J. L., Bommi-Reddy, A., Girnun, G., et al. (2008). HIF-independent regulation of VEGF and angiogenesis by the transcriptional a topic for future research to expand our knowledge of myokine regulation at the transcriptional level.

\section{CONCLUSION}

An "exercise pill" to reproduce the multiple beneficial effects of exercise on various organs seems to be unpractical because exercise affects too many interconnected pathways and its holistic effects also depend on the host genetics. Nonetheless, expanding our knowledge about which types of activity in humans control beneficial myokine secretion could give valuable, more personalized suggestions to patients about which kind of exercise to practice. Selected myokine-based drugs could be given to act on specific pathways and help patients who for various reasons cannot exercise.

\section{AUTHOR CONTRIBUTIONS}

The author confirms being the sole contributor of this work and has approved it for publication. RP conceptualized the manuscript, reviewed the literature, and wrote the manuscript in its entirety.

\section{FUNDING}

This work was supported by Italian Association for Cancer Research (AIRC-IG 19927).

\section{ACKNOWLEDGMENTS}

I am grateful to the Italian Association for Cancer Research (AIRC-IG 19927) for financial support and I thank J. D. Baggott for help with the editing.

coactivator PGC-1alpha. Nature 451, 1008-1012. doi: 10.1038/nature 06613

Aversa, Z., Bonetto, A., Penna, F., Costelli, P., Di Rienzo, G., Lacitignola, A., et al. (2012). Changes in myostatin signaling in non-weight-losing cancer patients. Ann. Surg. Oncol. 19, 1350-1356. doi: 10.1245/s10434-011-1720-5

Bamberger, A.-M., Jenatschke, S., Schulte, H. M., Ellebrecht, I., Beil, F. U., and Bamberger, C. M. (2004). Regulation of the human leukemia inhibitory factor gene by ETS transcription factors. Neuroimmunomodulation 11, 10-19. doi: $10.1159 / 000072964$

Banzet, S., Koulmann, N., Sanchez, H., Serrurier, B., Peinnequin, A., and Bigard, A. X. (2007). Musclin gene expression is strongly related to fast-glycolytic phenotype. Biochem. Biophys. Res. Commun. 353, 713-718. doi: 10.1016/j.bbrc. 2006.12.074

Bazgir, B., Salesi, M., Koushki, M., and Amirghofran, Z. (2015). Effects of eccentric and concentric emphasized resistance exercise on IL-15 serum levels and its relation to inflammatory markers in athletes and non-athletes. Asian J. Sports Med. 6:e27980. doi: 10.5812/asjsm.27980

Benatti, F. B., and Pedersen, B. K. (2015). Exercise as an anti-inflammatory therapy for rheumatic diseases-myokine regulation. Nat. Rev. Rheumatol. 11, 86-97. doi: 10.1038/nrrheum.2014.193 
Besse-Patin, A., Montastier, E., Vinel, C., Castan-Laurell, I., Louche, K., Dray, C., et al. (2014). Effect of endurance training on skeletal muscle myokine expression in obese men: identification of apelin as a novel myokine. Int. J. Obes. 38, 707-713. doi: 10.1038/ijo.2013.158

Bloch, S. A. A., Lee, J. Y., Wort, S. J., Polkey, M. I., Kemp, P. R., and Griffiths, M. J. D. (2013). Sustained elevation of circulating growth and differentiation factor-15 and a dynamic imbalance in mediators of muscle homeostasis are associated with the development of acute muscle wasting following cardiac surgery. Crit. Care Med. 41, 982-989. doi: 10.1097/CCM.0b013e318 $274671 \mathrm{~b}$

Bobadilla, M., Sainz, N., Abizanda, G., Orbe, J., Rodriguez, J. A., Páramo, J. A., et al. (2014). The CXCR4/SDF1 axis improves muscle regeneration through MMP-10 activity. Stem Cells Dev. 23, 1417-1427. doi: 10.1089/scd.2013.0491

Boström, P., Wu, J., Jedrychowski, M. P., Korde, A., Ye, L., Lo, J. C., et al. (2012). A PGC1-alpha-dependent myokine that drives brown-fat-like development of white fat and thermogenesis. Nature 481, 463-468. doi: 10.1038/nature 10777

Broholm, C., Mortensen, O. H., Nielsen, S., Akerstrom, T., Zankari, A., Dahl, B., et al. (2008). Exercise induces expression of leukaemia inhibitory factor in human skeletal muscle. J. Physiol. 586, 2195-2201. doi: 10.1113/jphysiol.2007. 149781

Catoire, M., and Kersten, S. (2015). The search for exercise factors in humans. FASEB J. 29, 1615-1628. doi: 10.1096/fj.14-263699

Catoire, M., Mensink, M., Kalkhoven, E., Schrauwen, P., and Kersten, S. (2014). Identification of human exercise-induced myokines using secretome analysis. Physiol. Genomics 46, 256-267. doi: 10.1152/physiolgenomics. 00174.2013

Carson, B. P. (2017). The potential role of contraction-induced myokines in the regulation of metabolic function for the prevention and treatment of Type 2 diabetes. Front. Endocrinol. 8:97. doi: 10.3389/fendo.2017.00097

Chung, H. S., Hwang, S. Y., Choi, J. H., Lee, H. J., Kim, N. H., Yoo, H. J., et al. (2018). Implications of circulating Meteorin-like (Metrnl) level in human subjects with type 2 diabetes. Diabetes Res. Clin. Pract. 136, 100-107. doi: 10.1016/j.diabres.2017.11.031

Ciciliot, S., Rossi, A. C., Dyar, K. A., Blaauw, B., and Schiaffino, S. (2013). Muscle type and fiber type specificity in muscle wasting. Int. J. Biochem. Cell Biol. 45, 2191-2199. doi: 10.1016/j.biocel.2013.05.016

Costelli, P., Muscaritoli, M., Bonetto, A., Penna, F., Reffo, P., Bossola, M., et al. (2008). Muscle myostatin signalling is enhanced in experimental cancer cachexia. Eur. J. Clin. Invest. 38, 531-538. doi: 10.1111/j.1365-2362.2008.01970.x

Dalla Libera, L., Ravara, B., Volterrani, M., Gobbo, V., Della Barbera, M., Angelini, A., et al. (2004). Beneficial effects of GH/IGF-1 on skeletal muscle atrophy and function in experimental heart failure. Am. J. Physiol. Cell Physiol. 286, C138-C144.

Demontis, F., Piccirillo, R., Goldberg, A. L., and Perrimon, N. (2013a). Mechanisms of skeletal muscle aging: insights from Drosophila and mammalian models. Dis. Model. Mech. 6, 1339-1352. doi: 10.1242/dmm.012559

Demontis, F., Piccirillo, R., Goldberg, A. L., and Perrimon, N. (2013b). The influence of skeletal muscle on systemic aging and lifespan. Aging Cell 12, 943-949. doi: 10.1111/acel.12126

Dong, J., Dong, Y., Dong, Y., Chen, F., Mitch, W. E., and Zhang, L. (2016). Inhibition of myostatin in mice improves insulin sensitivity via irisin-mediated cross talk between muscle and adipose tissues. Int. J. Obes. 40, 434-442. doi: 10.1038/ijo.2015.200

Dray, C., Knauf, C., Daviaud, D., Waget, A., Boucher, J., Buleon, M., et al. (2008). Apelin stimulates glucose utilization in normal and obese insulin-resistant mice. Cell Metab. 8, 437-445. doi: 10.1016/j.cmet.2008.10.003

Eaton, M., Granata, C., Barry, J., Safdar, A., Bishop, D., and Little, J. P. (2018). Impact of a single bout of high-intensity interval exercise and short-term interval training on interleukin-6, FNDC5, and METRNL mRNA expression in human skeletal muscle. J. Sport Health Sci. 7, 191-196. doi: 10.1016/j.jshs. 2017.01.003

Egerman, M. A., Cadena, S. M., Gilbert, J. A., Meyer, A., Nelson, H. N., Swalley, S. E., et al. (2015). GDF11 increases with age and inhibits skeletal muscle regeneration. Cell Metab. 22, 164-174. doi: 10.1016/j.cmet. 2015.05.010
Fearon, K., Strasser, F., Anker, S. D., Bosaeus, I., Bruera, E., Fainsinger, R. L., et al. (2011). Definition and classification of cancer cachexia: an international consensus. Lancet Oncol. 12, 489-495. doi: 10.1016/S1470-2045(10)70218-7

Flück, M., and Hoppeler, H. (2003). Molecular basis of skeletal muscle plasticityfrom gene to form and function. Rev. Physiol. Biochem. Pharmacol. 146, 159-216. doi: 10.1007/s10254-002-0004-7

Furrer, R., and Handschin, C. (2018). Muscle wasting diseases: novel targets and treatments. Annu. Rev. Pharmacol. Toxicol. 59, 315-339. doi: 10.1146/annurevpharmtox-010818-021041

Gannon, N. P., Vaughan, R. A., Garcia-Smith, R., Bisoffi, M., and Trujillo, K. A. (2015). Effects of the exercise-inducible myokine irisin on malignant and nonmalignant breast epithelial cell behavior in vitro. Int. J. Cancer 136, E197-E202. doi: 10.1002/ijc. 29142

Gao, X., and Xu, Z. (2008). Mechanisms of action of angiogenin. Acta Biochim. Biophys. Sin. 40, 619-624. doi: 10.1111/j.1745-7270.2008.00442.x

Hambrecht, R., Schulze, P. C., Gielen, S., Linke, A., Möbius-Winkler, S., Yu, J., et al. (2002). Reduction of insulin-like growth factor-I expression in the skeletal muscle of noncachectic patients with chronic heart failure. J. Am. Coll. Cardiol. 39, 1175-1181. doi: 10.1016/S0735-1097(02) 01736-9

Han, H. Q., Zhou, X., Mitch, W. E., and Goldberg, A. L. (2013). Myostatin/activin pathway antagonism: molecular basis and therapeutic potential. Int. J. Biochem. Cell Biol. 45, 2333-2347. doi: 10.1016/j.biocel.2013.05.019

Hawley, J. A., Hargreaves, M., Joyner, M. J., and Zierath, J. R. (2014). Integrative biology of exercise. Cell 159, 738-749. doi: 10.1016/j.cell.2014.10.029

He, Z., Tian, Y., Valenzuela, P. L., Huang, C., Zhao, J., Hong, P., et al. (2018). Myokine response to high-intensity interval vs. resistance exercise: an individual approach. Front. Physiol. 9:1735. doi: 10.3389/fphys.2018.01735

Hittel, D. S., Axelson, M., Sarna, N., Shearer, J., Huffman, K. M., and Kraus, W. E. (2010). Myostatin decreases with aerobic exercise and associates with insulin resistance. Med. Sci. Sports Exerc. 42, 2023-2029. doi: 10.1249/MSS. 0b013e3181e0b9a8

Hojman, P., Dethlefsen, C., Brandt, C., Hansen, J., Pedersen, L., and Pedersen, B. K. (2011). Exercise-induced muscle-derived cytokines inhibit mammary cancer cell growth. Am. J. Physiol. Endocrinol. Metab. 301, E504-E510. doi: 10.1152/ ajpendo.00520.2010

Hulmi, J. J., Silvennoinen, M., Lehti, M., Kivelä, R., and Kainulainen, H. (2012). Altered REDD1, myostatin, and Akt/mTOR/FoxO/MAPK signaling in streptozotocin-induced diabetic muscle atrophy. Am. J. Physiol. Endocrinol. Metab. 302, E307-E315. doi: 10.1152/ajpendo.00398.2011

Ijiri, N., Kanazawa, H., Asai, K., Watanabe, T., Hirata, K., and Irisin, A. (2015). newly discovered myokine, is a novel biomarker associated with physical activity in patients with chronic obstructive pulmonary disease. Respirology 20, 612-617. doi: 10.1111/resp.12513

Ishiuchi, Y., Sato, H., Komatsu, N., Kawaguchi, H., Matsuwaki, T., Yamanouchi, K., et al. (2018). Identification of CCL5/RANTES as a novel contraction-reducible myokine in mouse skeletal muscle. Cytokine 108, 17-23. doi: 10.1016/j.cyto. 2018.03.012

Izumiya, Y., Bina, H. A., Ouchi, N., Akasaki, Y., Kharitonenkov, A., and Walsh, K. (2008a). FGF21 is an Akt-regulated myokine. FEBS Lett. 582, 3805-3810. doi: 10.1016/j.febslet.2008.10.021

Izumiya, Y., Hopkins, T., Morris, C., Sato, K., Zeng, L., Viereck, J., et al. (2008b). Fast/Glycolytic muscle fiber growth reduces fat mass and improves metabolic parameters in obese mice. Cell Metab. 7, 159-172. doi: 10.1016/j.cmet.2007.11.003

Jia, D., Cai, M., Xi, Y., Du, S., and Zhenjun, T. (2018). Interval exercise training increases LIF expression and prevents myocardial infarction-induced skeletal muscle atrophy in rats. Life Sci. 193, 77-86. doi: 10.1016/j.lfs.2017.12.009

Ju, C.-R., and Chen, R.-C. (2012). Serum myostatin levels and skeletal muscle wasting in chronic obstructive pulmonary disease. Respir. Med. 106, 102-108. doi: 10.1016/j.rmed.2011.07.016

Kalantar-Zadeh, K., Rhee, C., Sim, J. J., Stenvinkel, P., Anker, S. D., and Kovesdy, C. P. (2013). Why cachexia kills: examining the causality of poor outcomes in wasting conditions. J. Cachexia Sarcopenia Muscle 4, 89-94. doi: 10.1007/ s13539-013-0111-0

Kanzleiter, T., Rath, M., Gorgens, S. W., Jensen, J., Tangen, D. S., Kolnes, A. J., et al. (2014). The myokine decorin is regulated by contraction and involved 
in muscle hypertrophy. Biochem. Biophys. Res. Commun. 450, 1089-1094. doi: 10.1016/j.bbrc.2014.06.123

Kim, H.-J., So, B., Choi, M., Kang, D., and Song, W. (2015). Resistance exercise training increases the expression of irisin concomitant with improvement of muscle function in aging mice and humans. Exp. Gerontol. 70, 11-17. doi: 10.1016/j.exger.2015.07.006

Kim, J.-S., Lee, Y.-H., and Yi, H.-K. (2016). Gradual downhill running improves age-related skeletal muscle and bone weakness: implication of autophagy and bone morphogenetic proteins. Exp. Physiol. 101, 1528-1540. doi: 10.1113/ EP085852

Kotler, D. P., Tierney, A. R., Wang, J., and Pierson, R. N. (1989). Magnitude of body-cell-mass depletion and the timing of death from wasting in AIDS. Am. J. Clin. Nutr. 50, 444-447. doi: 10.1093/ajcn/50.3.444

Lach-Trifilieff, E., Minetti, G. C., Sheppard, K., Ibebunjo, C., Feige, J. N., Hartmann, S., et al. (2014). An antibody blocking activin type II receptors induces strong skeletal muscle hypertrophy and protects from atrophy. Mol. Cell Biol. 34, 606-618. doi: 10.1128/MCB.01307-13

Laker, R. C., Garde, C., Camera, D. M., Smiles, W. J., Zierath, J. R., Hawley, J. A., et al. (2017). Transcriptomic and epigenetic responses to short-term nutrient-exercise stress in humans. Sci. Rep. 7:15134. doi: 10.1038/s41598-017-15420-7

Larsson, L., Degens, H., Li, M., Salviati, L., Lee, Y. I., Thompson, W., et al. (2019). Sarcopenia: aging-related loss of muscle mass and function. Physiol. Rev. 99, 427-511. doi: 10.1152/physrev.00061.2017

Lecker, S. H., Jagoe, R. T., Gilbert, A., Gomes, M., Baracos, V., Bailey, J., et al. (2004). Multiple types of skeletal muscle atrophy involve a common program of changes in gene expression. FASEB J. 18, 39-51. doi: 10.1096/fj.030610com

Lozano, R., Naghavi, M., Foreman, K., Lim, S., Shibuya, K., Aboyans, V., et al. (2012). Global and regional mortality from 235 causes of death for 20 age groups in 1990 and 2010: a systematic analysis for the Global Burden of Disease Study 2010. Lancet 380, 2095-2128. doi: 10.1016/S0140-6736(12)61728-0

Ma, W. Y., Yu, T. Y., Wei, J. N., Hung, C. S., Lin, M. S., Liao, Y. J., et al. (2014). Plasma apelin: a novel biomarker for predicting diabetes. Clin. Chim. Acta 435, 18-23. doi: $10.1016 /$ j.cca.2014.03.030

Mammucari, C., Milan, G., Romanello, V., Masiero, E., Rudolf, R., Del Piccolo, P., et al. (2007). FoxO3 controls autophagy in skeletal muscle in vivo. Cell Metab. 6, 458-471. doi: 10.1016/j.cmet.2007.11.001

Man, W. D.-C., Natanek, S. A., Riddoch-Contreras, J., Lewis, A., Marsh, G. S., Kemp, P. R., et al. (2010). Quadriceps myostatin expression in COPD. Eur. Respir. J. 36, 686-688. doi: 10.1183/09031936.00032510

Manole, E., Ceafalan, L. C., Popescu, B. O., Dumitru, C., and Bastian, A. E. (2018). Myokines as possible therapeutic targets in cancer cachexia. J. Immunol. Res. 2018:8260742. doi: 10.1155/2018/8260742

Marklund, P., Mattsson, C. M., Wåhlin-Larsson, B., Ponsot, E., Lindvall, B., Lindvall, L., et al. (2013). Extensive inflammatory cell infiltration in human skeletal muscle in response to an ultraendurance exercise bout in experienced athletes. J. Appl. Physiol. 114, 66-72. doi: 10.1152/japplphysiol.01538.2011

Martinelli, G. B., Olivari, D., Re Cecconi, A. D., Talamini, L., Ottoboni, L., Lecker, S. H., et al. (2016). Activation of the SDF1/CXCR4 pathway retards muscle atrophy during cancer cachexia. Oncogene 35, 6212-6222. doi: 10.1038/onc. 2016.153

Mashili, F. L., Austin, R. L., Deshmukh, A. S., Fritz, T., Caidahl, K., Bergdahl, K., et al. (2011). Direct effects of FGF21 on glucose uptake in human skeletal muscle: implications for type 2 diabetes and obesity. Diabetes. Metab. Res. Rev. 27, 286-297. doi: 10.1002/dmrr.1177

McCall, G. E., Allen, D. L., Haddad, F., and Baldwin, K. M. (2003). Transcriptional regulation of IGF-I expression in skeletal muscle. Am. J. Physiol. Cell Physiol. 285, C831-C839. doi: 10.1152/ajpcell.00047.2003

McPherron, A. C., Lawler, A. M., and Lee, S. J. (1997). Regulation of skeletal muscle mass in mice by a new TGF-beta superfamily member. Nature $387,83-90$. doi: $10.1038 / 387083 \mathrm{a} 0$

McPherron, A. C., and Lee, S. J. (1997). Double muscling in cattle due to mutations in the myostatin gene. Proc. Natl. Acad. Sci. U.S.A. 94, 12457-12461. doi: 10. 1073/pnas.94.23.12457

McPherron, A. C., and Lee, S.-J. (2002). Suppression of body fat accumulation in myostatin-deficient mice. J. Clin. Invest. 109, 595-601. doi: 10.1172/JCI0213562
Munoz, R. M., Han, H., Tegeler, T., Petritis, K., Von Hoff, D. D., and Hoffman, S. A. (2013). Isolation and characterization of muscle fatigue substance with anti-tumor activities. J. Cancer 4, 343-349. doi: 10.7150/jca.5418

Nakamori, M., Hamanaka, K., Thomas, J. D., Wang, E. T., Hayashi, Y. K., Takahashi, M. P., et al. (2017). Aberrant myokine signaling in congenital myotonic dystrophy. Cell Rep. 21, 1240-1252. doi: 10.1016/j.celrep.2017.10.018

Nakamura, K., Yamanouchi, K., and Nishihara, M. (2014). Secreted protein acidic and rich in cysteine internalization and its age-related alterations in skeletal muscle progenitor cells. Aging Cell 13, 175-184. doi: 10.1111/acel.12168

Ostrowski, K., Schjerling, P., and Pedersen, B. K. (2000). Physical activity and plasma interleukin-6 in humans-effect of intensity of exercise. Eur. J. Appl. Physiol. 83, 512-515. doi: 10.1007/s004210000312

Passey, S. L., Hansen, M. J., Bozinovski, S., McDonald, C. F., Holland, A. E., and Vlahos, R. (2016). Emerging therapies for the treatment of skeletal muscle wasting in chronic obstructive pulmonary disease. Pharmacol. Ther. 166, 56-70. doi: 10.1016/j.pharmthera.2016.06.013

Pedersen, B. K., and Febbraio, M. A. (2008). Muscle as an endocrine organ: focus on muscle-derived interleukin-6. Physiol. Rev. 88, 1379-1406. doi: 10.1152/ physrev. 90100.2007

Pedersen, B. K., and Febbraio, M. A. (2012). Muscles, exercise and obesity: skeletal muscle as a secretory organ. Nat. Rev. Endocrinol. 8, 457-465. doi: 10.1038/ nrendo.2012.49

Penna, F., Bonetto, A., Muscaritoli, M., Costamagna, D., Minero, V. G., Bonelli, G., et al. (2010). Muscle atrophy in experimental cancer cachexia: is the IGF-1 signaling pathway involved? Int. J. Cancer 127, 1706-1717. doi: 10.1002/ijc. 25146

Piccirillo, R., Demontis, F., Perrimon, N., and Goldberg, A. L. (2014). Mechanisms of muscle growth and atrophy in mammals and Drosophila. Dev. Dyn. 243, 201-215. doi: 10.1002/dvdy.24036

Pilegaard, H., Saltin, B., and Neufer, P. D. (2003). Exercise induces transient transcriptional activation of the PGC-1alpha gene in human skeletal muscle. J. Physiol. 546, 851-858. doi: 10.1113/jphysiol.2002.034850

Pistilli, E. E., Devaney, J. M., Gordish-Dressman, H., Bradbury, M. K., Seip, R. L., Thompson, P. D., et al. (2008). Interleukin-15 and interleukin-15R alpha SNPs and associations with muscle, bone, and predictors of the metabolic syndrome. Cytokine 43, 45-53. doi: 10.1016/j.cyto.2008.04.008

Plant, P. J., Brooks, D., Faughnan, M., Bayley, T., Bain, J., Singer, L., et al. (2010). Cellular markers of muscle atrophy in chronic obstructive pulmonary disease. Am. J. Respir. Cell Mol. Biol. 42, 461-471. doi: 10.1165/rcmb.2008-0 $382 \mathrm{OC}$

Pretto, F., Ghilardi, C., Moschetta, M., Bassi, A., Rovida, A., Scarlato, V., et al. (2015). Sunitinib prevents cachexia and prolongs survival of mice bearing renal cancer by restraining STAT3 and MuRF-1 activation in muscle. Oncotarget 6, 3043-3054. doi: 10.18632/oncotarget.2812

Prontera, P., Bernardini, L., Stangoni, G., Capalbo, A., Rogaia, D., Ardisia, C., et al. (2009). 2q31.2q32.3 deletion syndrome: report of an adult patient. Am. J. Med. Genet. A 149A, 706-712. doi: 10.1002/ajmg.a.32688

Puchert, M., Adams, V., Linke, A., and Engele, J. (2016). Evidence for the involvement of the CXCL12 system in the adaptation of skeletal muscles to physical exercise. Cell. Signal. 28, 1205-1215. doi: 10.1016/j.cellsig. 2016.05.019

Quinn, L. S., Anderson, B. G., Strait-Bodey, L., and Wolden-Hanson, T. (2010). Serum and muscle interleukin-15 levels decrease in aging mice: correlation with declines in soluble interleukin-15 receptor alpha expression. Exp. Gerontol. 45, 106-112. doi: 10.1016/j.exger.2009.10.012

Rai, M., and Demontis, F. (2016). Systemic nutrient and stress signaling via myokines and myometabolites. Annu. Rev. Physiol. 78, 85-107. doi: 10.1146/ annurev-physiol-021115-105305

Roubenoff, R., and Kehayias, J. J. (1991). The meaning and measurement of lean body mass. Nutr. Rev. 49, 163-175. doi: 10.1111/j.1753-4887.1991.tb03013.x

Rovira, M., Arrey, G., and Planas, J. V. (2017). Exercise-induced hypertrophic and oxidative signaling pathways and myokine expression in fast muscle of adult zebrafish. Front. Physiol. 8:1063. doi: 10.3389/fphys.2017.01063

Rutti, S., Dusaulcy, R., Hansen, J. S., Howald, C., Dermitzakis, E. T., Pedersen, B. K., et al. (2018). Angiogenin and Osteoprotegerin are type II muscle specific myokines protecting pancreatic beta-cells against proinflammatory cytokines. Sci. Rep. 8:10072. doi: 10.1038/s41598-018-28117-2 
Safdar, A., and Tarnopolsky, M. A. (2018). Exosomes as mediators of the systemic adaptations to endurance exercise. Cold Spring Harb. Perspect. Med. 8:a029827. doi: 10.1101/cshperspect.a029827

Sandri, M., Lin, J., Handschin, C., Yang, W., Arany, Z. P., Lecker, S. H., et al. (2006). PGC-1alpha protects skeletal muscle from atrophy by suppressing FoxO3 action and atrophy-specific gene transcription. Proc. Natl. Acad. Sci. U.S.A. 103, 16260-16265. doi: 10.1073/pnas.0607795103

Sandri, M., Sandri, C., Gilbert, A., Skurk, C., Calabria, E., Picard, A., et al. (2004). Foxo transcription factors induce the atrophy-related ubiquitin ligase atrogin1 and cause skeletal muscle atrophy. Cell 117, 399-412. doi: 10.1016/S00928674(04)00400-3

Schiaffino, S., and Serrano, A. (2002). Calcineurin signaling and neural control of skeletal muscle fiber type and size. Trends Pharmacol. Sci. 23, 569-575. doi: 10.1016/S0165-6147(02)02111-9

Seldin, M. M., Peterson, J. M., Byerly, M. S., Wei, Z., and Wong, G. W. (2012). Myonectin (CTRP15), a novel myokine that links skeletal muscle to systemic lipid homeostasis. J. Biol. Chem. 287, 11968-11980. doi: 10.1074/jbc.M111. 336834

Seto, D. N., Kandarian, S. C., and Jackman, R. W. (2015). A key role for leukemia inhibitory factor in C26 cancer cachexia. J. Biol. Chem. 290, 19976-19986. doi: 10.1074/jbc.M115.638411

Shah, R., Hinkle, C. C., Ferguson, J. F., Mehta, N. N., Li, M., Qu, L., et al. (2011). Fractalkine is a novel human adipochemokine associated with type 2 diabetes. Diabetes 60, 1512-1518. doi: 10.2337/db10-0956

Shin, K. O., Bae, J. Y., Woo, J., Jang, K. S., Kim, K. S., Park, J. S., et al. (2015). The effect of exercise on expression of myokine and angiogenesis mRNA in skeletal muscle of high fat diet induced obese rat. J. Exerc. Nutr. Biochem. 19, 91-98. doi: 10.5717/jenb.2015.15061006

Simonet, W. S., Lacey, D. L., Dunstan, C. R., Kelley, M., Chang, M. S., Lüthy, R., et al. (1997). Osteoprotegerin: a novel secreted protein involved in the regulation of bone density. Cell 89, 309-319. doi: 10.1016/S0092-8674(00) 80209-3

Son, J. S., Chae, S. A., Testroet, E. D., Du, M., and Jun, H.-P. (2018). Exerciseinduced myokines: a brief review of controversial issues of this decade. Expert Rev. Endocrinol. Metab. 13, 51-58. doi: 10.1080/17446651.2018.1416290

Subbotina, E., Sierra, A., Zhu, Z., Gao, Z., Koganti, S. R., Reyes, S., et al. (2015). Musclin is an activity-stimulated myokine that enhances physical endurance. Proc. Natl. Acad. Sci. U.S.A. 112, 16042-16047. doi: 10.1073/pnas.1514250112

Tanimura, Y., Aoi, W., Takanami, Y., Kawai, Y., Mizushima, K., Naito, Y., et al. (2016). Acute exercise increases fibroblast growth factor 21 in metabolic organs and circulation. Physiol. Rep. 4:e12828. doi: 10.14814/phy2.12828

Terada, S., Goto, M., Kato, M., Kawanaka, K., Shimokawa, T., and Tabata, I. (2002). Effects of low-intensity prolonged exercise on PGC-1 mRNA expression in rat epitrochlearis muscle. Biochem. Biophys. Res. Commun. 296, 350-354. doi: 10.1016/S0006-291X(02)00881-1

Tezze, C., Romanello, V., Desbats, M. A., Fadini, G. P., Albiero, M., Favaro, G., et al. (2017). Age-associated loss of OPA1 in muscle impacts muscle mass, metabolic homeostasis, systemic inflammation, and epithelial senescence. Cell Metab. 25, 1374-1389.e6. doi: 10.1016/j.cmet.2017.04.021

Toloza, F. J. K., Mantilla-Rivas, J. O., Pérez-Matos, M. C., Ricardo-Silgado, M. L., Morales-Alvarez, M. C., Pinzón-Cortés, J. A., et al. (2018). Plasma levels of myonectin but not myostatin or fibroblast-derived growth factor 21 are associated with insulin resistance in adult humans without diabetes mellitus. Front. Endocrinol. 9:5. doi: 10.3389/fendo.2018.00005
Trayhurn, P., Drevon, C. A., and Eckel, J. (2011). Secreted proteins from adipose tissue and skeletal muscle - adipokines, myokines and adipose/muscle cross-talk. Arch. Physiol. Biochem. 117, 47-56. doi: 10.3109/13813455.2010. 535835

VanderVeen, B. N., Fix, D. K., and Carson, J. A. (2017). Disrupted skeletal muscle mitochondrial dynamics, mitophagy, and biogenesis during cancer cachexia: a role for inflammation. Oxid. Med. Cell. Longev. 2017:3292087. doi: 10.1155/ 2017/3292087

Vinel, C., Lukjanenko, L., Batut, A., Deleruyelle, S., Pradère, J.-P., Le Gonidec, S., et al. (2018). The exerkine apelin reverses age-associated sarcopenia. Nat. Med. 24, 1360-1371. doi: 10.1038/s41591-018-0131-6

Walker, K. S., Kambadur, R., Sharma, M., and Smith, H. K. (2004). Resistance training alters plasma myostatin but not IGF-1 in healthy men. Med. Sci. Sports Exerc. 36, 787-793. doi: 10.1249/01.MSS.0000126384.04 778.29

Wang, Q., Guo, T., Portas, J., and McPherron, A. C. (2015). A soluble activin receptor type IIB does not improve blood glucose in streptozotocin-treated mice. Int. J. Biol. Sci. 11, 199-208. doi: 10.7150/ijbs.10430

Welle, S., Bhatt, K., Shah, B., and Thornton, C. (2002). Insulin-like growth factor-1 and myostatin mRNA expression in muscle: comparison between 62-77 and 21-31 yr old men. Exp. Gerontol. 37, 833-839. doi: 10.1016/S0531-5565(02)00025-6

Whitham, M., and Febbraio, M. A. (2016). The ever-expanding myokinome: discovery challenges and therapeutic implications. Nat. Rev. Drug Discov. 15, 719-729. doi: 10.1038/nrd.2016.153

Whitham, M., Parker, B. L., Friedrichsen, M., Hingst, J. R., Hjorth, M., Hughes, W. E., et al. (2018). Extracellular vesicles provide a means for tissue crosstalk during exercise. Cell Metab. 27, 237-251.e4. doi: 10.1016/j.cmet.2017.12.001

Winbanks, C. E., Chen, J. L., Qian, H., Liu, Y., Bernardo, B. C., Beyer, C., et al. (2013). The bone morphogenetic protein axis is a positive regulator of skeletal muscle mass. J. Cell Biol. 203, 345-357. doi: 10.1083/jcb.201211134

Winick, M. (1979). Hunger Disease-Studies by Jewish Physicians in the Warsaw Ghetto. New York, NY: John Wiley \& Sons.

Zhao, J., Brault, J. J., Schild, A., Cao, P., Sandri, M., Schiaffino, S., et al. (2007). FoxO3 coordinately activates protein degradation by the autophagic/lysosomal and proteasomal pathways in atrophying muscle cells. Cell Metab. 6, 472-483. doi: 10.1016/j.cmet.2007.11.004

Zhao, X., and Karpac, J. (2017). Muscle directs diurnal energy homeostasis through a myokine-dependent hormone module in Drosophila. Curr. Biol. 27, 1941-1955.e6. doi: 10.1016/j.cub.2017.06.004

Zhou, X., Wang, J. L., Lu, J., Song, Y., Kwak, K. S., Jiao, Q., et al. (2010). Reversal of cancer cachexia and muscle wasting by ActRIIB antagonism leads to prolonged survival. Cell 142, 531-543. doi: 10.1016/j.cell.2010.07.011

Conflict of Interest Statement: The author declares that the research was conducted in the absence of any commercial or financial relationships that could be construed as a potential conflict of interest.

Copyright (c) 2019 Piccirillo. This is an open-access article distributed under the terms of the Creative Commons Attribution License (CC BY). The use, distribution or reproduction in other forums is permitted, provided the original author(s) and the copyright owner(s) are credited and that the original publication in this journal is cited, in accordance with accepted academic practice. No use, distribution or reproduction is permitted which does not comply with these terms. 